\title{
Automated Behavioral Phenotype Detection and Analysis Using Color-Based Motion Tracking
}

\author{
Alan Shimoide $^{1} \quad$ Ilmi Yoon $^{1} \quad$ Megumi Fuse $^{2} \quad$ Holly C. Beale $^{2^{*}} \quad$ Rahul Singh $^{1}$ \\ alans@sfsu.edu $\quad$ yoon@,cs.sfsu.edu fuse@,sfsu.edu hollyb@caltech.edu rsingh@,cs.sfsu.edu \\ ${ }^{1}$ Computer Science Department, San Francisco State University
${ }^{2}$ Biology Department, San Francisco State University
}

Abstract

The problem of elucidating the functional significance of genes is a key challenge of modern science. Solving this problem can lead to fundamental advancements across multiple areas such starting from pharmaceutical drug discovery to agricultural sciences. A commonly used approach in this context involves studying genetic influence on model organisms. These influences can be expressed at behavioral, morphological, anatomical, or molecular levels and the expressed patterns are called phenotypes. Unfortunately, detailed studies of many phenotypes, such as the behavior of an organism, is highly complicated due to the inherent complexity of the phenotype pattern and because of the fact that it may evolve over long time periods. In this paper, we propose applying color-based tracking to study Ecdysis in the hornworm - a biologically highly relevant phenotype whose complexity had thus far, prevented application of automated approaches. We present experimental results which demonstrate the accuracy of tracking and phenotype determination under conditions of complex body movement, partial occlusions, and body deformations. A key additional goal of our paper is to expose the computer vision community to such novel applications, where techniques from vision and pattern analysis can have a seminal influence on other branches of modern science.

Keywords: Automated Phenotyping, Color-based Tracking, Gene function elucidation, Deformable object tracking, Spatio-temporal pattern analysis, ecdysis.

\section{Introduction}

In the biological sciences, one of the main ways to understand the functional significance of a gene is to determine the influence of specific gene products (such as proteins) on the organism in which the gene occurs. A powerful approach in this context involves studying the genetic influence at molecular, anatomical, morphological, and/or behavioral levels, using simple, well studied model organisms such as Drosophila melanogaster (fruit fly), Danio rerio (zebrafish), Man- duca sexta (hornworm), and the nematode Caenorhabditis elegans. Within this methodological framework, the problem of understanding the relationship between genes and behavior represents a fundamental challenge in neurosciences. Specific solutions to this problem can have applications in areas ranging from drug discovery to agricultural to disease control.

The elucidation of gene-behavior relationships using this integrated approach has two significant advantages. First, central as well as peripheral inputs to the nervous system can be assessed by comparing invasive and isolated in vitro responses with non-invasive behavioral correlates. Second, information generated from multiple model organisms can yield single scientific principles. For instance, ecdysis, or the shedding of the old cuticle, is a common behavior in arthropods such as insects, suggesting that studies conducted on $D$. melanogaster and M. sexta can be used to form a common model for the general regulation of ecdysis. Using state-of-the-art techniques in molecular genetics in $D$. melanogaster, it is possible to interact with and manipulate specific genes associated with ecdysis [McNabb97]. Thus molecular correlates of ecdysis are studied in vivo and in the context of an intact behavioral response. In contrast, the nervous system can be easily isolated and studied in M. sexta [Weeks84]. Thus, neural correlates at the anatomical level are correlated to similar ecdysis behaviors. Unfortunately however, the design of experiments based on behavioral phenotypes requires studying complex aspects of the selected model organism's behavior such as locomotion patterns, egg-laying, or ecdysis. The challenges involved in doing so include:

- Temporal complexity of observations: Often, behavioral phenotypes are expressed over time. However, the attention span of researchers observing the model organism is restricted due to fatigue. Therefore, the probability of observing and accurately describing behavioral phenotypes that are delayed in occurrence or develop over time is low. This in turn has the potential to preclude from consideration important gene-function relationships. Moreover, the rate at which studies can be done by these methods is low.

- Complexity of intra-phenotype determination: Even in the presence of well-defined phenotype

\footnotetext{
${ }^{2 *}$ Currently at: Division of Biology, California Institute of Technology
} 
categories, many behavioral phenotypes are only subtle alterations of one another. Such distinctions are difficult to detect reliably by eye, even for experienced observers. The overlay of multiple simultaneously occurring behaviors increases the detection issues. The problem is further complicated given the constraint that the decision needs to be made in real time.

These challenges demonstrate the strong need for quantitative measures, and for classification and analysis of behavioral phenotypes using robust computer vision methods. Tracking and analysis of the data may vary and should make best use of the domain knowledge for each organism, in order to integrate our understanding of the mechanisms of each organism within a unified scientific framework.

Relatively recently, approaches have been developed to track model organisms such as $C$. elegans [Baek02, Geng04] and rodents [Branson03], to automatically determine an assortment of behavioral phenotypes. In [Branson03], for instance, the tracking problem is considered in the context of mice. The paper proposes an optical flow-based approach for geometric (shape-based) tracking. For C. elegans, the problem of automated phenotyping has been researched in [baek02, geng04]. The tracking approach proposed in these studies is based on image binarization (conversion of the image to black-and-white) followed by derivation of geometric descriptors such as shape skeletons. In a supervised setting, a set of features are then determined for each phenotype, and a classification and regression tree (CART) algorithm is applied to come up with a decision tree, which can be used to classify observed phenotypes into known classes.

This paper presents the development of an algorithm to track behaviors of the hornworm, Manduca sexta, during periods of pre-ecdysis and ecdysis behaviors. Analysis and visualization of the behaviors are discussed in comparison with existing biological data, and in the context of a broader multi-organismal framework.

We begin by describing the significance of research on $M$. sexta, and the difficulties associated with tracking ecdysis behaviors in section 2 . The proposed

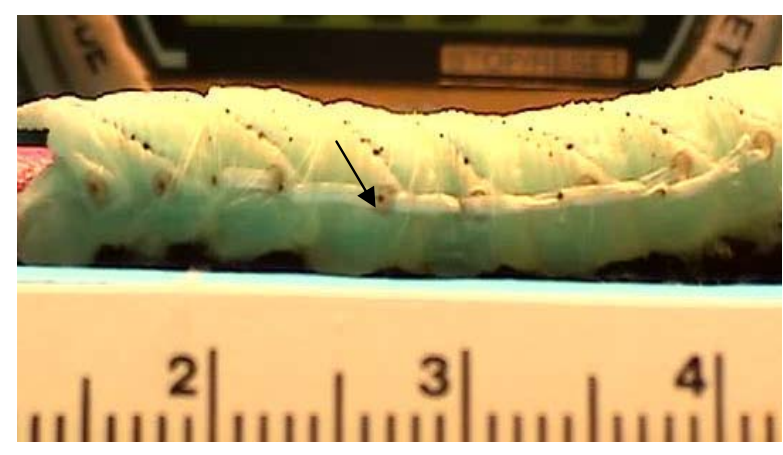

Figure 1 - A frame from Maduca sexta video clips spiracles are covered by cuticle during Ecdysis. Arrow points at a spiracle. approach is presented in section 3 followed by results in section 4. The paper is concluded in Section 5 with a synopsis of the current research and possible future directions..

\section{Challenges in Automated Phenotyping of Ecdysis in the hornworm}

\subsection{Importance of Ecdysis}

As insects grow they must periodically shed their outer shell, or cuticle, in order to continue growing and developing successfully. This process is termed ecdysis, and consists of a series of behaviors that include muscle contractions to loosen and eventually slough off the old cuticle. The insect is vulnerable at this point, and success depends on very tight coordination within the central and peripheral nervous systems to induce a rapid and precisely-timed ecdysis. In the hornworm, Manduca sexta, ecdysis consists of a well-characterized and stereotyped behavioral repertoire that can be visualized at the level of the whole animal as well as in single cells of the isolated nervous system. Thus, the neural control of ecdysis should be a simple and strong model for understanding the neural control of behaviors in general, and may prove important in understanding how the nervous system is modulated to improve flexibility or plasticity of behaviors in an everchanging environment.

Neuromodulation is essential for complex neural processing in humans, including perception and memory [Robbins2000], and its disruption is associated with diseases such as Parkinson's or Alzheimer's [Muchowski05]. A role for neuromodulators in addiction has also been considered [Fattore04], and companies have begun to appear which design, develop, manufacture and market advanced implantable neuromodulation devices [web-ref1].

The discrete stereotyped behaviors that define ecdysis consist of (i) synchronous muscle contractions along the segmented body wall (termed pre-ecdysis) followed by (ii) asynchronous or peristaltic contractions starting at the back of the animal and moving forward in a wave-like motion segment by segment (termed ecdysis). These behaviors have been studied at the muscular and cellular levels. However, whole animal ecdysis behaviors have only been analyzed by eye to a limited extent, and at present, researchers lack methodology to quantitatively describe them.

M. sexta has spiracles associated with each segment (Figure 1), which move with the body wall during the anteriorly-directed muscle contractions. Movement of these spiracles has been shown to correlate with the onset of ecdysis behaviors, suggesting that spiracle movement is a good measure of muscle contractions related to ecdysis [Beale03]. The changes in distance between any two spiracles define the extent of muscle contraction or relaxation, and this occurs for each thoracic and abdominal segment. Thus, if spiracles are robustly tracked, then not only are the muscle contractions quantified within a segment, but also the 


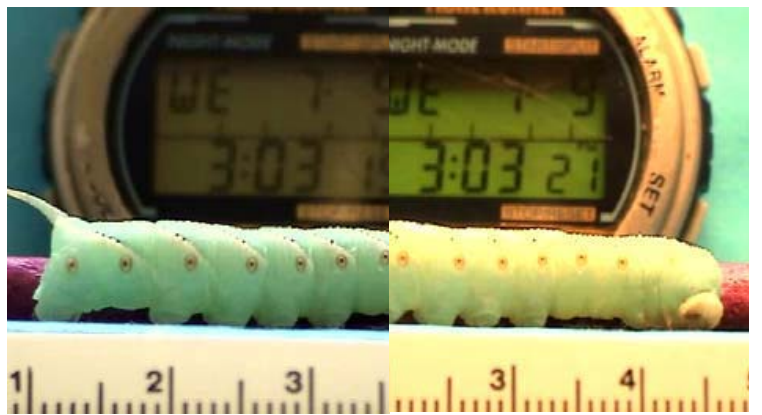

Figure 2 - Radical shading changes within 2 or 3 frames

interactions between segments can be analyzed. Outputs associated with modifications to the nervous system (eg. brain lesions) can be easily quantified in this way. More importantly, researchers will be able to determine if the burst patterns of recordings from isolated nerves that provide the triggers for muscle contractions (called fictive ecdysis behaviors) are correlated with the behavioral output measured by our tracking program. These results should determine whether peripheral inputs that are lost with dissection of the nervous system are essential for modulating the final output or not. That is, if the frequency of contractions of the animal's body differs from the frequency of bursting of the isolated nerves, it is probable that peripheral inputs to the nervous system, which are lost after dissection, are critical in modulating the final behavioral output.

\subsection{Complexity of Monitoring Ecdysis}

The spiracles in insects are openings along the body wall that enable air flow through a tracheal system designed to transport oxygen to the body. The trachea are cuticularly lined, and so tracheal cuticle is sloughed off with the rest of the body cuticle during ecdysis. Thus, the spiracles are often partially or entirely masked by tracheal cuticle emerging from its opening (Figure 1), or from the more anterior spiracle, at points during the behavioral repertoire. This makes tracking challenging. Moreover, to successfully ecdyse, $M$. sexta is videotaped unrestrained, and often moves considerably from the starting position. Researchers often refocus the camera, change the camera magnification, manually shift the position of the organism while taping or place the organism in front of complex backgrounds. Moreover, most biological settings fluctuate heavily, where radical shading changes or zooming in and out is common. Shifting of the camera occurs frequently during a video sequence and hands can obstruct views. Obviously these changes in animal position, magnification, complex backgrounds and lighting (Figure 2) increase the difficulties in sustained tracking.

Object segmentation or tracking from video has been an active research area in the vision and image processing community. Within much of the literature, automatic segmentation usually assumes that the object can be described as either the moving region or the region that moves with consistent parameters [Choi97, [Altunbasak98]. For those objects that cannot be easily tracked automatically, semantics of objects and multiple features are utilized to segment and track objects [Gu98, Castango98]. For example, [Toklu00] used an active mesh to represent and track objects. Further, tracking object boundaries based on neighboring color and motion information has be attemped in [Gu98, Fu00, and Luo99]. While our approach shares ideas with the aforementioned research, the problem we address is more complex. For example, the cuticles often occlude the spiracles during ecdysis, making it difficult to determine their boundaries. It is also challenging to track the geometry of the whole organism during ecdysis since the shape of the organism changes dramatically as it undergoes muscle contractions.

\section{Proposed Methodology \\ 3.1. Tracking}

The ability to track and assess the whole animal movements non-invasively is essential for understanding the final output of neural signaling that produces repertoires of behaviors seen in animals. To robustly track Manduca through the entire ecdysis process using non-invasive methods, we only need an initial user input to choose the overall the body size and the locations of individual spiracles (Figure 3). We take advantage of a characteristic of $M$. sexta, namely that while the muscle contraction deforms body shape, the relation of the spiracles to the body remains invariant, to robustly track the spiracles during ecdysis or preecdysis. This invariant allows effective handling of problems associated with sudden camera movements or variations in shading.

To be able to extract the overall shape of the hornworm from the background, we use a 3D color space. Based on the user's initial input, we distinguish the body of the organism (Body) as the area contained in the bounding box and the body background (BodyBackground) asthe area surrounding the bounding box (Figure 4). Distinct color groups are created for the body and body background using a 3D histogram of the color space. To account for noise and changes in lighting, a color tolerance for the Body ( $\triangle \mathrm{BTol})$ representing a specified distance from each color in $3 \mathrm{D}$ space is used. Colors within the color tolerance are considered to be similar to each other. The color group for the Body Background is determined by using a histogram and ranking the colors to determine the

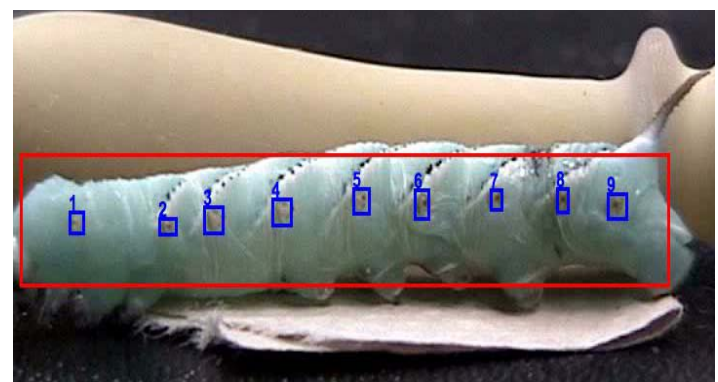

Figure 3 - User's initial input. Spiracles are incrementally numbered by their positions. 


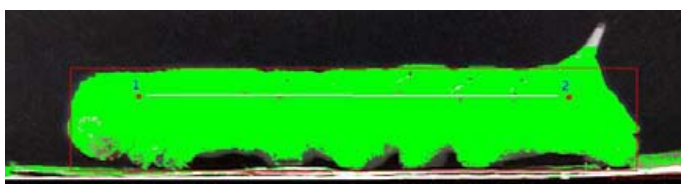

Figure 4 - the body color group defined by using the 3D color histogram.

prominent colors of the BodyBackground (CBodyBack). From this initial color group, CBodyBack, we subtract a subset of colors from the Body (CBody). This susbset of colors is determined using aminimal bounding box of the body.

$$
\begin{array}{r}
\text { BodyBackground Color }=\text { CBodyBack }- \\
\subseteq(C B o d y \pm \Delta B T o l)
\end{array}
$$

The color group representing the Body is determined in a similar fashion except that we distinguish between highly prominent colors in the Body (HighCBody) and colors that are not so prominent in the Body (LowCBody). Colors that are not so prominent are only used if they differ greatly from the colors in the Body Background. The LowCBody colors can represent colors at the border of the Body and colors at the border can be similar to the Background. To ensure that the LowCBody colors differ greatly from the Body Background we aggressively remove any color from the LowCBody if that color is within user controlled parameter $(\mathrm{x})$ multiplied with the normal Body color tolerance of the Body Background.

$$
\begin{array}{r}
\text { Body Color }=(\text { HighCBody }-(\text { CBodyBack } \pm \text { BBTol }))+ \\
(\text { LowCBody }-(\text { CBodyBack } \pm x \Delta B T o l))
\end{array}
$$

If the Body cannot be distinguished from the Body Backgroud, i.e. they are the same color, then:

$$
\text { Body Background }=\varnothing
$$

The color group representing the spiracles (Feature) and the area surrounding the spiracles (Feature Background) is also determined using a histogram of the $3 \mathrm{D}$ color space. The area surrounding each spiracle is determined using the user's bounding box of the spiracle, the size of the Body, and location of the spiracle within the Body. Colors within the area of the Feature Background are ranked and the most prominent ones are used to determine the color ground for the Feature Background (CFeatureBack).

Feature Background Color $=$ CfeatureBack

The Feature color group is determined by using a minimal bounding box for each spiracle. A color tolerance for the Features $(\Delta \mathrm{FTol})$, similar to the one in the Body is used. Colors within the Feature are ranked for prominence and colors that belong to the Feature Background are removed. We distinguish between colors of high (HighCFeat) and low prominence (LowCFeat) in the Feature.

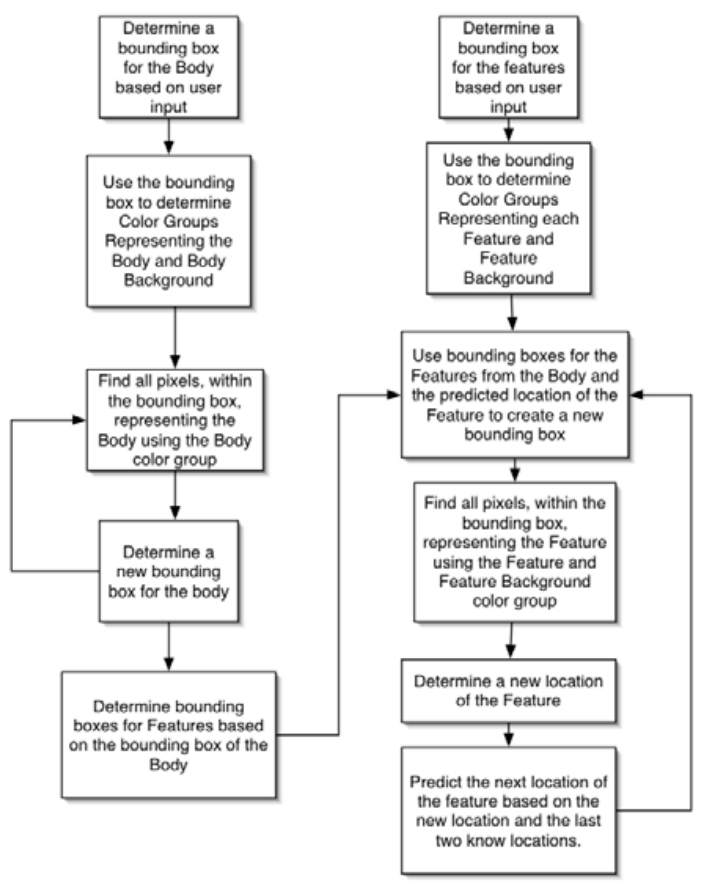

Figure 5 - Algorithm for Body and Feature tracking

Feature Color $=($ HighCFeat $-($ CFeatureBack $\pm \Delta F T o l))$

$+($ LowCFeat $-($ CFeatureBack $\pm 3 x \Delta F T o l))$

If the Feature cannot be distinguished from the Feature Background then:

Feature Background $=\varnothing$

Once all color groups have been determined, the shape of the Body is extracted based on its color group. Spatial coherence is used to find pixels representing the Body by searching within a bounding box based on the last known location of the Body. The shape of the organism is found by finding a new bounding box for the pixels in the Body color group that were found. Pixels matching the Body are found using the Body color tolerance. If the color of the pixel (Cpixel) matches a color from the Body color group (CBody) it is considered to be a Body Pixel.

Body Pixel $=$ Cpixel $\cap($ CBody $\pm \Delta$ Btol $)$

When using only color, tracking may become very sensitive to noise and changes in lighting. Noise in the video with colors similar to the spiracle would create false results, regardless of the size and shape of the noise. Simple color tracking cannot distinguish one spiracle from another. Color tracking also has difficulties in handling the cuticle of $M$. sexta that covers its spiracles during ecdysis. Just as the cuticle occludes the shape of the spiracle, much of the color information is also not visible (Figure 1). Therefore, the temporal coherence and spatial coherence are properly employed to update color scheme to robustly track spiracles during radical lighting changes. This enabled a 
robust and accurate tracking system that handles changes in the environment.

Spiracles are tracked using spatial and temporal coherence, also. Spatial coherence of the spiracles is utilized by using the predicted bounding area, basesd on the body, for each spiracle, and temporal coherence is utilized by using the last known location of the spiracle to predict the new location of the spiracle. A bounding box for the spiracle is calculated using the last known predicted location and the predicted area from the body.

\section{PredictedLocation $=$ LastLocation $-($ oldLocation + oldOldLocation) $/ 2+$ LastLocation}

Pixels representing the spiracles are determined by finding pixels within this bounding box that match the color group of the Feature. If the color of the pixel (CPixel) is a color in the Feature color group (CFeature) we check to see if the surrounding pixels belong to the Feature background. To reduce interference from noise in the video, the color of the pixels neighboring this pixel are analyzed $\left(\mathrm{CNeighPixel}_{1-8}\right)$ to see if they belong to the Feature Background. If the neighboring pixels are not from the Feature background (!(CNeighPixel ${ }_{1-8}$ $\cap$ CFeatureBack $\pm \Delta$ Ftol)) then this pixel is a pixel representing the feature. Feature Pixel $=$ Cpixel $\cap($ Cfeature $\pm \Delta$ Ftol $)$
$!\left(\right.$ CNeighPixel $_{1-8} \cap$ CFeatureBack $\pm \Delta$ Ftol $)$

The new location of the Feature is calculated based on the calculated location (CalcLocation) and the last know location of the Feature (LastLocation) and a confidence factor (ConfFact). The calculated location of the Feature, CalcLocation, is based on either an average of the location of all pixels found or on a calculated center of the Feature based on the size and shape of the Feature, from the pixels found, whichever is closer to Last Location. The calculated center (CalcCenter) finds a bounding box for the largest cluster of pixels representing the feature that was found. If too many clusters of pixels are found, such as when the cuticle partially covers the spiracle, an average of the location of all pixels would be better and is used.

\section{If(AvgLocation is closer to LastLocation) \{ CalcLocation $=$ AvgLocation of pixels \} else if (CalcCenter is closer to LastLocation) \{ CaclcLocation $=$ CalcCenter of pixels \}}

To calculate the new location of the Feature, a confidence factor is used. The confidence factor correlates directly with the number of pixels representing the feature that were found. The confidence factor is higher if there were more pixels found and lower if there were less pixels found. For example, if no pixels representing the feature was found then the confidence factor would be zero and the Location for the Feature would be the LastLocation. If there were many pixels found then the ConfFact would be much higher and
LastLocation would influence the new Location of the Feature less. The main steps of the algorithm are shown in Figure 5.

Location of Feature $=($ ConfFact $x$ CalcLocation +
LastLocation $) /($ ConfFact +1$)$

\subsection{Analysis and Visualization}

Successful tracking of the location of spiracles

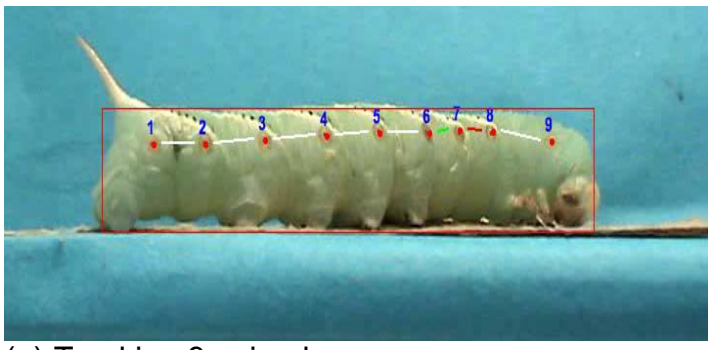

(a) Tracking 9 spiracles.

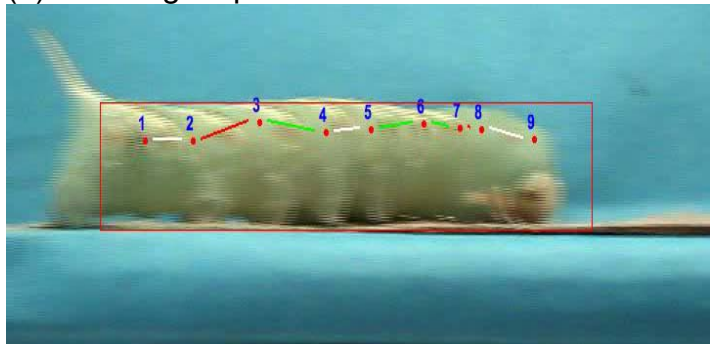

(b) Camera is being moved radically.



(c) Spiracles are lost.



(d) The body position is recalculated.



(e) The program has fully recovered.

Figure $\mathbf{6}$ - Tracking recovers from radical camera shift. 
opens up many opportunities to analyze multiple sets of data quantitatively, and to handle massive numbers of clips for accurate statistics and classification. We first assess changes in distance of spiracles on the body as a measure of muscle contractions. To enhance the user's grasp of the information while watching tracking, lines between each spiracle are visualized in green and red to represent the contraction status in real time. For instance, green and red can represent contracted or relaxed muscle states, respectively. The distance between the spiracles is also plotted graphically in real time (Figure.8), showing the frequency and amplitude of contractions, for all segments. Rates of change of these values can further be assessed under different experimental conditions. These values can be normalized relative to body size of the animal to make samples comparable to one another.

This data will provide a quantitative measure of behaviors that are typically only qualitatively described in the literature. Behavioral changes after experimental manipulations such as brain surgeries are then statistically comparable between groups. More importantly, researchers will be able to determine whether the burst patterns of recordings from isolated nerves, that are the triggers for the muscle contractions producing the behaviors, (called fictive ecdysis bursts) are directly correlated with the behavioral outputs measured by our tracking program. This may help de-



(a) Image shows tracking is successful during the ecdysis
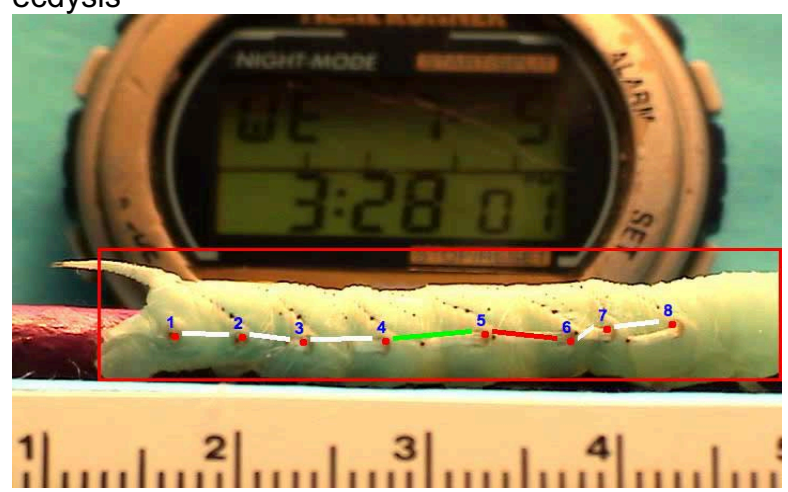

(c) Within the same second, the contraction segments move from (b) 6-8 to (c) 4-6. termine whether there are peripheral inputs to the nervous system and muscles, that are lost with dissection of the nervous system from the body, add a level of complexity to modulation of the final output. That is, if the frequency of contractions of the animal's body differs from the frequency of bursting of the isolated nerves, one can assume that peripheral inputs to the nervous system, which are lost after dissection, are critical in modulating the final behavioral output.

\section{Experimental Evaluations and Results}

The Feature tracking algorithms were implemented in Java, using QuickTime for Java to handle the videos. Various video clips with tracking are available at "http://unicorn.sfsu.edu/ tracking". The results are discussed in terms of (i) accuracy of tracking, (ii) robustness in handling visual blurring during cuticle shedding or other environments, (iii) execution time and (iv) confirmation with hand-measured data or fictive burst patterns.

\subsection{Accuracy}

To gauge the accuracy of the Feature tracking algorithms, the location of spiracles were tracked in several videos using the Java implementation of the algorithms and the tracking results were monitored for errors. Misplacement of one or more features is defined as an error. The accuracy of tracking videos is discussed under three categories of video clips: videos with no

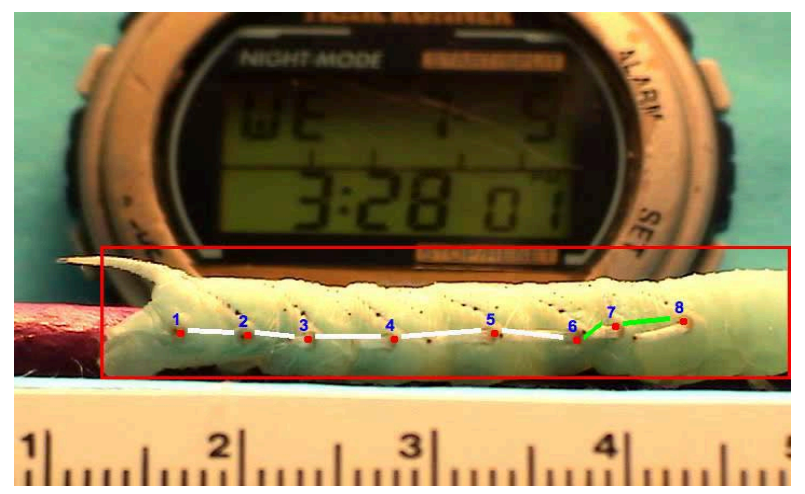

(b) Ecdysis is still in progress (34 frames after (a)) and tracking is successful.

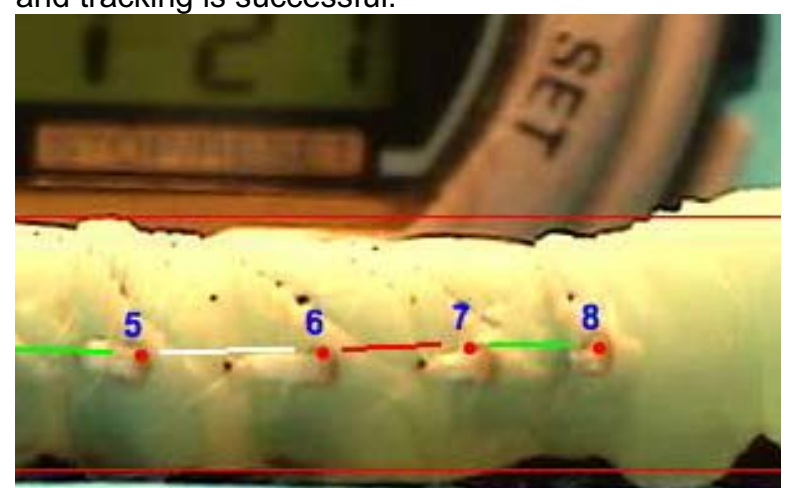

(d) Spiracles become visually unclear, but tracking is performed successfully.

Figure 7 - Tracking is robustly handled during ecdysis. 
significant noise, videos with significant noise, and videos with the cuticle partially or wholly covering the spiracles. Videos with no significant noise imply that there was no radical camera movement, rolling of the organism, manipulation of the organism, or lighting changes in the video. Though these videos can be analyzed manually, it is tedious, and very time-consuming

Videos without significant noise: Out of approximately 53000 tracked features (5880 frames x 9 spiracles), there were about 150 features tracked with errors. This results in a $99.8 \%$ success rate. When calculated at a frame basis instead of an individual feature basis, out of 5880 frames, there were 105 frames with one or two features misplaced.

Most of the tracking errors were errors in tracking the location of one or two spiracles only. Data from frames with an error in tracking the location of one or two spiracles can be handled as outliers since the locations of the spiracles in nearby frames are correct.

Videos with significant noise: Out of 7350 frames analyzed, there were 1890 frames with significant noise. Out of the 66150 features (7350 frames x 9 spiracles) there were about 3000 features $(2100$ frames with one or two errors) with tracking errors. This resulted in a $95.5 \%$ success rate at a feature basis. Considering that $26 \%$ of the video frames had severe noise, our tracking system shows very quick recovery from the noisy frames. With this error rate, we can still use them in the analyses by utilizing the confidence level for each feature. When the confidence level assigned to each feature is low, then we can consider them as outliers, so they don't affect the final results.

Videos with the cuticle partially or wholly covering the spiracles: Out of 80460 features (8940 frames x 9 spiracles) analyzed there were about 3500 features with tracking errors (1860 frames with errors in tracking the location of two spiracles or less). This resulted in a

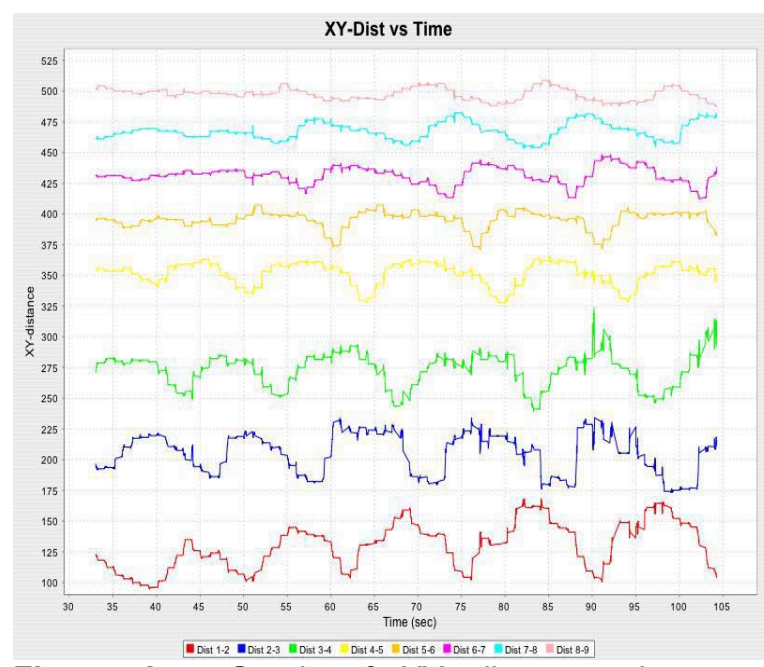

Figure 8 - Graph of $X Y$ distances between Spiracles vs Time, in seconds, during Ecdysis. 9 spiracles were tracked for 105 seconds and their $X Y$ distances, relative to each other, were graphed one on top of each other.
95.4\% success rate in tracking videos with the cuticle partially or wholly covering the spiracle.

In summary, the whole feature tracking success rate included about 6650 errors out of 199610 features, or $96.7 \%$. Most of all, our tracking showed robust and quick recovery from severely noisy frames without the need for human intervention as shown in figures 6 and 7 .

\subsection{Performance (speed)}

On a $1 \mathrm{GHz}$ machine, the Java Feature tracking program processed about 7 frames per second with an image size of $720 \times 480$, for tracking of 9 spiracles and a body, which provides interactive and real time tracking to users.

\subsection{Confirmation with hand-measured data}

To confirm whether or not the video tracking was properly measuring the defined behavioral output of M. sexta, nine features were tracked on 3750 frames of video and changes in their XY-distances were analyzed to see if the feature tracking correlated with results measured by eye (and manual tracking), and with results from fictive ecdysis bursts in the isolated nervous systems.

The frequency of muscle contractions was approximated, by measuring the distance between two peaks from the Feature tracking results, or from measurements of segmental contractions made by eye. These were compared, and were also compared to fictive ecdysis bursts (see Table 1). For the segment of ecdysis behaviors we analyzed, frequencies of contraction with computer tracking or measurements by eye are almost identical $(p<0.001)$. However, contractions differed significantly from fictive ecdysis bursts $(p>0.5)$. Thus, Feature Tracking properly measures the behavioral output of $M$. sexta but does not directly correlate with the fictive ecdysis bursts. This suggests that the role the nervous system plays in the regulation needs to be evaluated in the context of peripheral neuromodulatory influences. Feature Tracking results and fictive burst patterns will be compared during the course of ecdysis as well as under different experimental conditions in our continuing research.

\subsection{Analysis of results and future directions}

It is clear from the above example that Feature Tracking is a reliable method for quantifying ecdysis behaviors. This tracking can be used to monitor the onset of ecdysis, which will help identify modulators

\begin{tabular}{|l|l|l|}
\hline Tracking Results & Hand Results & Fictive Bursts \\
\hline $0.086 \pm 0.009$ & $0.086 \pm 0.005$ & $0.055 \pm 0.005$ \\
\hline
\end{tabular}

Table 1 - Average frequency of contraction and standard error of the mean for ecdysis contractions measured by Feature Tracking and by eye, as well as frequency of fictive ecdysis bursts measured electrophysiologically. Sample size was 5-11. 
that regulate aspects of the behaviors. Monitoring ecdysis was the basis for suggesting that inhibitors modulated timing of onset of ecdysis [Fuse02], but studies were limited to single segmental measurements. More importantly, Figure 8 shows that that multiple measurements can be determined, for each segmental behavior, simultaneously, offering a new quantitative approach to understanding the behaviors. For instance, it has always been assumed that fictive ecdysis bursts correlate with output, and that recordings from any two segmental nerves will define this. As seen in Figure 8, peak contraction amplitudes are not equal from segment to segment, although frequencies of contraction are (data not shown, $\mathrm{p}>0.5$ ), suggesting that different inputs will regulate the speed, strength and maybe even the duration of contractions in the hornworm. Feature Tracking will allow researchers to conduct an integrated analysis of all segments at once. It has been suggested that the strength and frequency of contractions changes during ecdysis, and again it will be important to not only determine this quantitatively, but to determine how this varies from segment to segment.

\section{Conclusion}

The ability to continuously track morphological features on the body without the need for preexisting external markers on the body has numerous applications such as the analysis of human gait, tracking of cell movements, analysis of animal behaviors in the wild and image synthesis for special effects. Our research extends applications of tracking to elucidating behavioral influences of genes. We have developed a color-based tracking approach to automatically detect, quantify, and present complex behavioral phenotypes (such as ecdysis) in M. sexta, a model organism that is extensively used in biological research. Results presented in this paper provide a view of the final behavioral output regulated by neural signaling that is often lost by the invasive nature of many contemporary scientific measurement techniques. We anticipate that our research will lead to new paradigms of highthroughput model-organism-based research involving unprecidented amounts of data. This will lead to discovery of behavioral patterns that have not been previously identified and lead to a deeper understanding of gene-behavior relationships.

\section{Acknowledgements}

We would like to thank Luping May and AlexVaughan for technical and intellectual contribution to this paper.

\section{References}

[Altunbasak98] Y. Altunbasak and A. M. Takalp, "Region-based parametric motion segmentation using color information", Graph. Models Image Process., 60(1):13-23, 1998.

[Baek02] J.-H. Baek, P. Cosman, Z. Feng, J. Silver, and W. R. Schafer, "Using Machine Vision to analyze and classify Caenorhabditis elegans behavioral phenotypes quantitatively", J. Neurosci. Methods, 118(1):9-21, 2002.
[Beale03] H. Beale, and M. Fuse, "Computer Analysis of Whole Animal Behaviors: A Novel Method of Analyzing Insect Ecdysis." Society for Neuroscience Conference Abstract, 2003.

[Branson03] K. Branson, V. Rabaud, and S. Belongie, "Three Brown Mice: See How They Run", VS-PETS, 2003.

[Castango98] R. Castango, T. Ebrahimi and M. Kunt, "Video Segmentation Based on Multiple Features for Interactive Multimedia Application", IEEE Trans. Circuits and Syst. Video Technol. 8(5): Sept. 1998.

[Choi97] J. G. Choi, S.W. Lee, and S. D. Kim, "Spatiotemporal video segmentation using a joint similarity measure," IEEE Trans. Circuits Syst. Video Technol., 7:279-285, 1997.

[Fattore04] L. Fattore, G. Cossu, M. S. Spano, S. Deiana, P. Fadda, M. Scherma, and W. Fratta, "Cannabinoids and reward: interactions with the opioid system", Crit Rev Neurobiol. 16(1-2):147-58, 2004.

[Fu00] Y. Fu, A. T. Erdem, and A. M. Tekalp, "Tracking visible boundary of objects using occlusion adaptive motion snake," IEEE Trans. Image Processing, 9:2051-2060, 2000 .

[Geng04] W. Geng, P. Cosman, C. C. Berry, Z. Feng, and W. R. Schafer, "Automatic tracking, Feature extraction and classification of C. elegans phenotypes" IEEE Transactions on Biomedical Engineering, 51:1811-20, 2004.

[Gu98] C. Gu and M. C. Lee, "Semiautomatic segmentation and tracking of semantic video objects," IEEE Trans. Circuits Syst. Video Technol., 8(5):572-84, 1998.

[Luo99] H. Luo and A. Eleftheriadis, "Designing an interactive tool for video object segmentation and annotation," ACM Int. Multimedia Conf., Orlando, FL, Oct. 1999.

[McNabb97] S. L. McNabb, J. D. Baker, J. Agapite, H. Steller, L. M. Riddiford, and J. W. Truman, "Disruption of a behavioral sequence by targeted death of peptidergic neurons in Drosophila" Neuron 19(4):813-23, 1997.

[Mesce02] K. Mesce, and S. Fahrbach, "Integration of Endocrine Signals That Regulate Insect Ecdysis" Frontiers in Neuroendocrinology 23:179-199, 2002.

[Muchowski05] P. J. Muchowski, and J. L. Wacker, "Modulation of neurodegeneration by molecular chaperones", Nat Rev Neurosci. 6(1):11-22, 2005.

[Robbins00]. T. W. Robbins, "Chemical neuromodulation of frontal-executive functions in humans and other animals" Exp Brain Res. 133(1):130$8,2000$.

[Toklu00] C. Toklu, A. M. Tekalp, and A. T. Erdem, "Semi-automatic alpha plane generation by occlusionadaptive mesh tracking," IEEE Trans. Circuits Syst. Video Technol., 10(4):624-629, 2000.

[Weeks84] J. C. Weeks, and J. W. Truman, "Neural organization of peptide-activated ecdysis behaviors during the metamorphosis of Manduca sexta. I. Conservation of the peristalsis motor pattern at the larval-pupa transformation", J. Comp. Physiol A 15:407-422, 1984. [web-ref1] http://www.ans-medical.com 